\title{
Palm Vein Verification System Based on SIFT Matching
}

\author{
Pierre-Olivier Ladoux ${ }^{1}$, Christophe Rosenberger ${ }^{2}$, and Bernadette Dorizzi ${ }^{1}$ \\ ${ }^{1}$ Institue TELECOM \\ Télécom \& Management SudParis \\ 9 Rue Charles Fourier, 91011 Évry Cedex, France \\ pierre-olivier.ladoux@it-sudparis.eu, \\ bernadette.dorizzi@it-sudparis.eu \\ ${ }^{2}$ Laboratoire GREYC \\ ENSICAEN - CNRS - Université de Caen Basse-Normandie \\ 6 boulevard Maréchal Juin, 14000 Caen, France \\ christophe.rosenberger@ensicaen.fr
}

\begin{abstract}
We present in this communication a new biometric system based on the use of hand veins acquired by an infrared imager. After the preprocessing stage and binarization, the vein image is characterized by specific patterns. One originality of the proposed system is to use SIFT descriptors for the verification process. The developed method only necessitates a single image for the enrollment step allowing a very fast verification. The experimental results on a database containing images of 24 individuals acquired after two sessions show the efficiency of the proposed method.
\end{abstract}

Keywords: Hand vein modality, IR acquisition, normalization preprocessing, SIFT descriptors, key points matching.

\section{Introduction}

Hand vein is a biometric modality that seems promising as it is acquired in Near Infrared light (NIR), which implies that skin variations and dirtiness are less sensible than in visible light [1]. Moreover, the hemoglobin which flows in the veins is sensible to NIR light, this way allowing a good quality of acquisition of the hand veins. It is possible to use either the back of the hand or the hand palm. A recent study [2] using back hand vein data and tested with 5 sessions per person and 50 persons showed promising results. The main problem of this database is the low resolution of the images (images at resolution $132 \times 124$ pixels).

The first commercialized products have been produced by Hitachi [3] on the back and Fujitsu [4] on the palm. They have been patented but only little information is available on them. These companies claim a very low FRR at very low FAR on a huge database - close to $0 \%$ on 140000 hands.

Unfortunately at this moment, there is no public database allowing verifying these figures. In general, in the various papers present in the literature, after the acquisition phase, some preprocessing algorithms are used such as histogram equalization, low pass filtering. Then, some binarization is performed and for verification using this 
image, two main streams can be found: global matching of the reference and test images through a pixel to pixel superposition [5] or after some frequency analysis as in Laplacian palm processing [8]. Another direction consists in performing local matching of specific feature points extracted in the two references and test images [6]. The matching algorithms in this last approach are similar to those used for fingerprint verification [7].

Our approach developed in this paper falls in the last category. However, we use for the matching of the feature points, the well known SIFT [9] algorithm which, to our knowledge has never been used so far for hand vein verification. This algorithm developed for graph matching was proven to be very efficient for face verification [10]. We tested the proposed system on a small home database of videos of the hand palm of 24 persons containing two sessions.

We present in Section 2 the details of our approach including a synopsis of the developed system, the acquisition device we used for acquiring the palm database as well as the preprocessing we made on these images. We also explain our binarization procedure. In the following subsection, the detection of the feature points and the SIFT procedure is briefly described. Due to the fact that some translations and rotations are present between the two sessions, we also propose a post processing allowing a better separation of genuine and impostor's scores. Finally, section 3 presents the experimental work, namely the protocols defined on each database and the results in terms of EER and ROC curves.

\section{Description of the Proposed System}

The proposed scheme of the proposed method is shown in Figure 1.

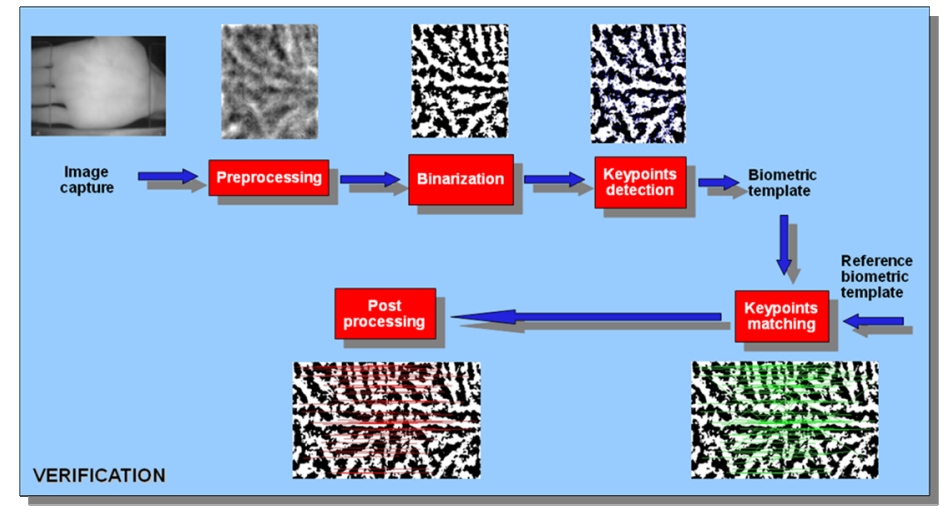

Fig. 1. Synopsis of the developed method (verification step)

It is standard in biometrics with an enrollment and a verification step. One important characteristic of our method is that only one image is needed for the enrollment step. Moreover, a post-processing is added after the matching phase in order to tackle translation and rotation problems. 


\subsection{Acquisition}

Image acquisition is done by 48 infrared LEDs and a CCD camera, which sensitive range is between $400 \mathrm{~nm}$ and $900 \mathrm{~nm}$ but the interesting wavelength is around $850 \mathrm{~nm}$. We added to this system one support in order to help the persons positioning their hand and to therefore limit translations and rotations. The hand is approximately at $20 \mathrm{~cm}$ of the camera's lens (see Figure 2).

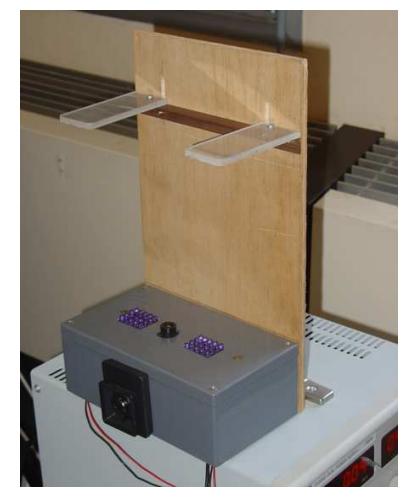

Fig. 2. NIR acquisition device

At each acquisition, we acquired a short video which provided us a set of 30 greyscale pictures of size $640 \times 320$ pixels. We this way acquired the data of 24 persons in two sessions.

\subsection{Pre-processing}

The first step of the pre-processing is the extraction of the region of interest (ROI). Due to limited translation and rotation, this is eased. Sizes are cut down to $232 \times 280$ pixels. Then, we apply the $5 \times 5$ box filter on the ROI in order to reduce the noise.

After removing the high frequency noise, we need to correct the brightness, which is not uniform. A Gaussian low-pass 51x51 filter is applied on the ROI in order to obtain the brightness image which is considered as low frequencies. Then, the brightness is subtracted of the original ROI.

At this step, the contrast is still too bad. We therefore apply a normalization method commonly used in fingerprint verification [7]. For each image I of size NxM, the mean and variance (denoted $\mu$ and $\sigma$ respectively) are calculated. Equation describes the normalization process applied on the image with $\mu_{d}$ and $\sigma_{d}$, the desired value of the mean and variance. For each pixel, we modify its gray-level with the following formula:

$$
I^{\prime}(x, y)= \begin{cases}\mu_{d}+\sqrt{\frac{\left.\sigma d^{2} \mathrm{~g} I(x, y)-\mu\right)^{2}}{\sigma^{2}}} & I(x, y)>\mu \\ \mu_{d}-\sqrt{\frac{\sigma d^{2} \mathrm{~g}(I(x, y)-\mu)^{2}}{\sigma^{2}}} & \text { Otherwise }\end{cases}
$$



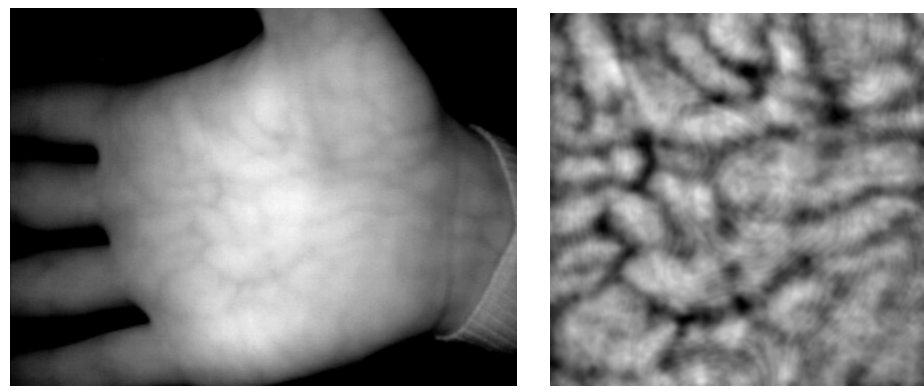

Fig. 3. (left) NIR image of palm, (right) Image after pre-processing

Where $I(x, y)$ corresponds to the gray-level for the pixel located at $(x, y)$ for the original image and $I^{\prime}(x, y)$ for the resulting one after pre-processing. Figure 3 shows the original image of the palm acquired with our sensor and the corresponding image after preprocessing. For our experiments, we set empirically the values of $\mu_{\mathrm{d}}$ to 128 and $\sigma_{\mathrm{d}}$ to $40^{2}$.

\subsection{Vein Pattern Extraction}

After noise reduction and contrast normalization, the quality of the image is improved as can be seen in Figure 3. To obtain the vein pattern, it is necessary to extract the veins from the background. In fact, the grey level is low where the hemoglobin absorbs the NIR. Therefore, the chosen extraction algorithm is a local thresholding one depending on the mean value of the neighborhood of each pixel.

Figure 4 shows two processing results for two images for the same individual. If we would ask somebody to decide if these images correspond to the same individual, he would probably try to find similar areas between these two images. This is the idea of the proposed methodology defined in the next section.
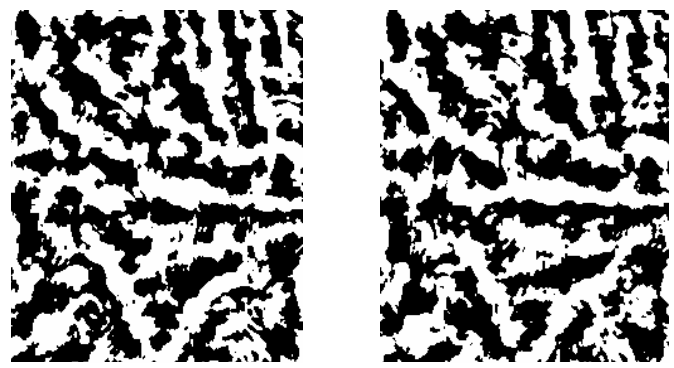

Fig. 4. Examples of two binarized images corresponding to the same individual

\subsection{Pattern Definition}

We chose to use a local description of the vein image in order to facilitate the verification step. We used the SIFT descriptor, following of a comparative study [11] showing that it is one of the most efficient local ones.

The invariant descriptor developed in the SIFT algorithm described in [9] is applied locally on key-points and is based on the image gradients in a local neighborhood. The 
SIFT detector and descriptor are constructed from the Gaussian scale space of the source image. The algorithm makes use of another scale space too, called difference of Gaussian (DOG), which can be considered as the scale derivative of the Gaussian scale space. Extracted key-points are defined as points of local extremum of the DOG scale space.

The descriptor is created by sampling the magnitudes and orientations of the image gradients in a neighborhood of each key-point and building smoothed orientation histograms that contain the important aspect of the neighborhood. Each local descriptor is composed on a $4 \times 4$ array (histogram). To each coordinate of this array, an 8 orientation vector is associated. A 128-elements vector is then built for each key-point.

We used in this article the implementation provided by Lowe [9]. As illustration, we obtained an average value of 800 detected key-points for the vein images at hand.

\subsection{Matching Similarity}

Each individual is described by a set of invariant features $Y(I)=\left\{k_{i}=\left(s_{i}, x_{i}, y_{i}\right)\right\}$, $i=1: N(I)$ where $s_{i}$ is the 128-elements SIFT invariant descriptor computed near keypoint $k_{i},\left(x_{i}, y_{i}\right)$ its position in the original image $I$ and $N(I)$ the number of detected keypoints for image $I$.

The verification problem for an individual given the set $Y(I)$ corresponding to the biometric model of an individual in our case, is to measure the similarity with another set of keypoints computed on the supposed vein image of the individual. We thus have to compute a similarity between two sets of points $Y\left(I_{1}\right)$ and $Y\left(I_{2}\right)$. We thus use the following matching method which is a modified version of a decision criterion first proposed by Lowe [9]:

Given two points $x \in Y\left(I_{1}\right)$ and $\mathrm{y} \in Y\left(I_{2}\right)$, we say that $x$ is associated to $y$ iff :

$$
d(x, y)=\min _{\{z \in Y(I 2)\}} d(x, z) \text { and } d(x, y) \leq C d\left(x, y^{\prime}\right)
$$

Where $C$ is an arbitrary threshold, $d(.,$.$) denotes the Euclidean distance between the$ SIFT descriptors and $y^{\prime}$ denotes any point of $Y\left(I_{2}\right)$ whose distance to $x$ is minimal but greater than $d(x, y)$ :

$$
d\left(x, y^{\prime}\right)=\min _{\{z \in Y(I 2), d(x, z)>d(x, y)\}} d(x, z)
$$

In other words, $x$ is associated to $y$ if $y$ is the closest point from $x$ in $Y\left(I_{2}\right)$ according to the Euclidean distance between SIFT descriptors and if the second smallest value of this distance $d\left(x, y^{\prime}\right)$ is significantly greater than $d(x, y)$. The significance of the necessary gap between $d(x, y)$ and $d\left(x, y^{\prime}\right)$ is encoded by the constant $C$. In the same way, we say that $y \in Y\left(I_{2}\right)$ is associated to $x \in Y\left(I_{1}\right)$ iff $x$ is the closest point from $y$ among $Y\left(I_{1}\right)$ according to the Euclidean distance between SIFT descriptors and if the second smallest value of this distance $d\left(y, x^{\prime}\right)$ satisfies $d(y, x)<C d\left(y, x^{\prime}\right)$. Then, we will say that $x$ is matched to $y$ iff $x$ is associated to $y$ and $y$ is associated to $x$. If $Y\left(I_{1}\right)$ and $Y\left(I_{2}\right)$ do not define any matching point, we consider that both sets have an infinite distance.

Figure 5 shows an example of matching results for an impostor and a genuine. We obtained 23 detected associations for an impostor (left result) and 63 for the genuine one. In the whole database, the average value for impostors equals 6 (we show in Figure 5 the comparison result having the larger number of associations for an impostor) and 30 for genuine ones.

The number of associations is used here as a similarity measure. 

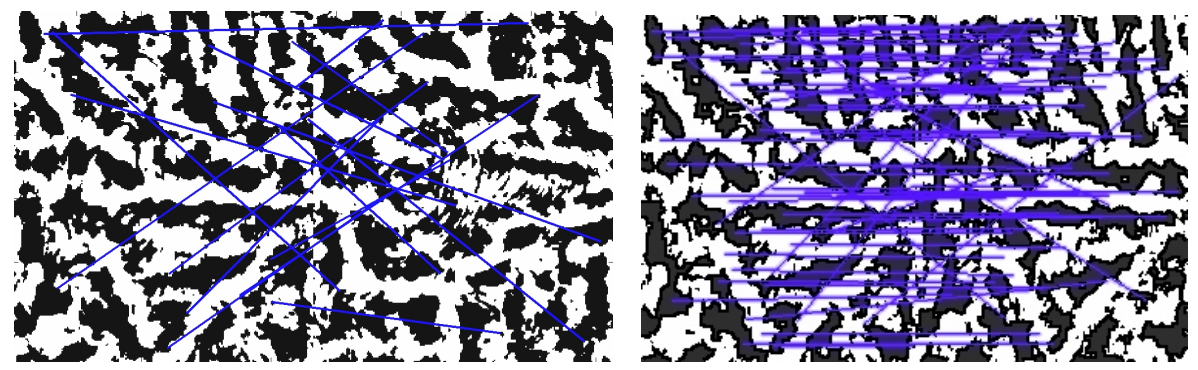

Fig. 5. Example of matching result for an impostor (on the left) and with a genuine (on the right)

\subsection{Post-processing}

The post-processing has for objective to select among the detected associations the relevant ones. The acquisition can provide mainly small translation and rotation artifacts. That means that the associations for genuine images corresponding to displacement vectors in Figure 5 shall be relatively collinear. In order to determine relevant ones, we compute the direction of each displacement vector by considering the keypoints location in each image to match. We compute the histogram of orientation of these vectors (with a step of 0.1 radian). The updated similarity value is the number of associations for the highest peak of the histogram.

Figure 6 shows the post-processing result of the association result given in Figure 5 (right). Note that we obtained a similarity value equals to 6 instead 23 and 51 instead 63 for the results presented in Figure 3. This shows that we significantly increase the difference between genuine and impostor samples.

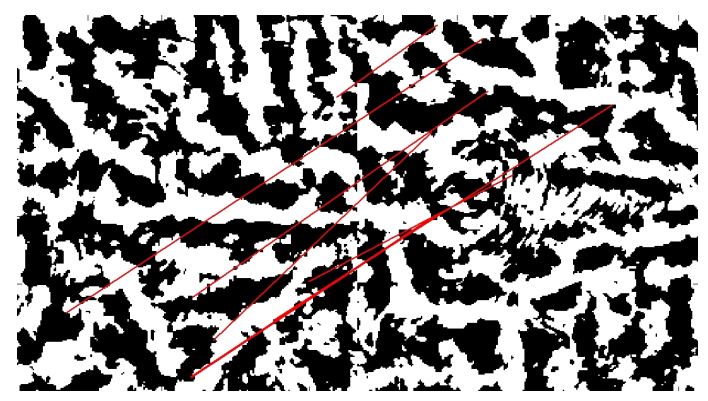

Fig. 6. Post processing of the result of Figure 5 (right)

\section{Experimental Results}

\subsection{Protocol}

We realized a home database with 24 individuals. Two sessions have been made and videos of hand have been acquired. We extracted from videos, 30 images for each session. The total amount of images used for this experiment is $24 \times 30 \times 2=1440$ images.

We use one image for the enrollment and 59 images for the test for each individual. 


\subsection{Performance Evaluation}

We used several measures to quantify the performance of the proposed system. In order to compute the False Rejection Rate (FRR) value, we compare the biometric reference with all samples of the same individual. For the False Acceptance Rate (FAR) value, we compare the biometric reference of an individual with all samples from different individuals.

For out test database, the FRR value is therefore computed considering $24 \times 59=$ 1416 matching procedures and the FAR value uses $24 \times 59 \times 23=32568$ ones. We will use the Equal Error Rate (EER) measure that corresponds to the value of the biometric system threshold for which FAR equals to FRR.

We will plot the disparities distribution between intraclass and interclass matching results and the ROC (Receiver Operating Characteristic) curve.
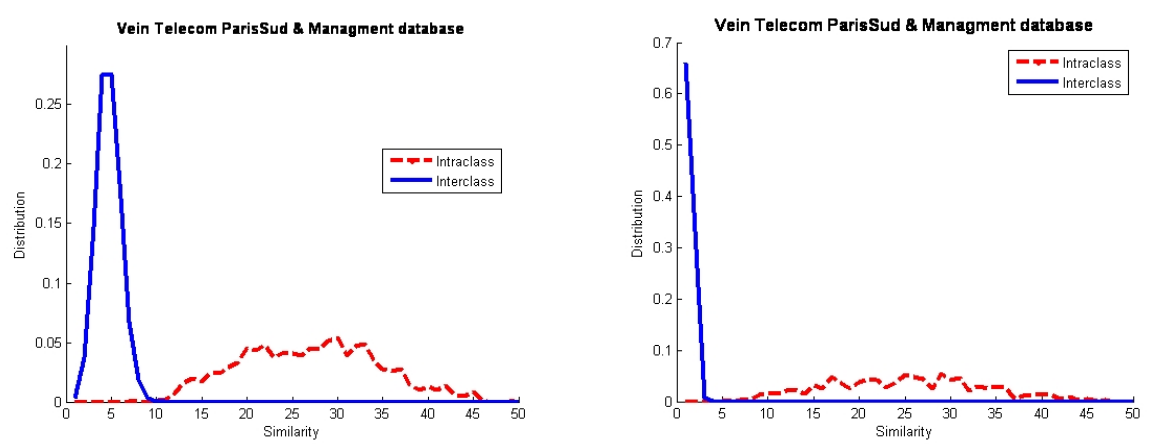

Fig. 7. Disparities distribution for the proposed method on the test database (left) without any post-processing (right) with post-processing

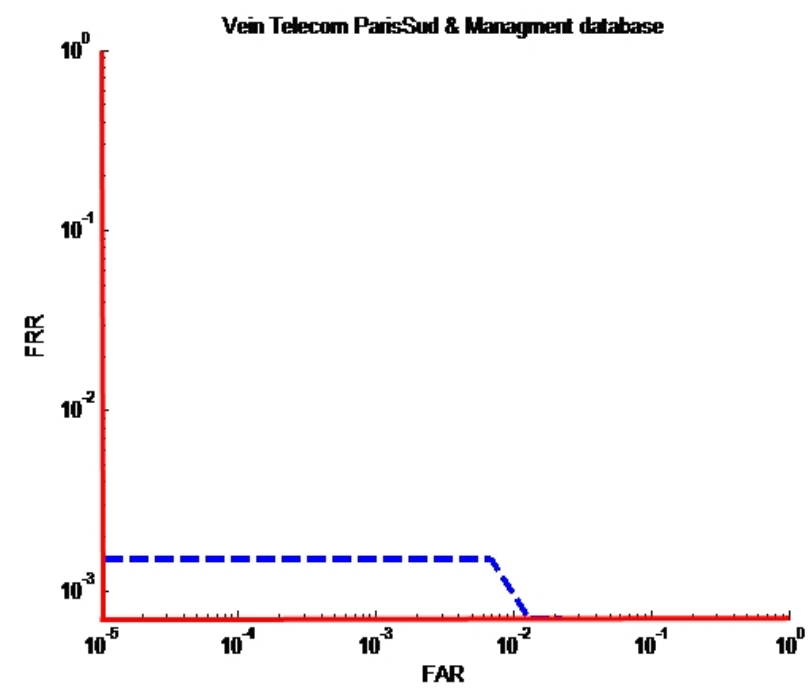

Fig. 8. DET curve of the proposed method without any post-processing (blue dotted line) and with processing (red line) 


\subsection{Results}

First, we plot the distribution of the intraclass and interclass disparities on the test database using the proposed method without and with post-processing. The benefit of the post-processing is clear. It allows a good separation between genuine and impostors.

Computing the DET curve of the proposed method without any post-processing (see Figure 8) we obtain an EER value equals to $0.14 \%$. After using the proposed post-processing, we obtained an ideal EER value that is to say $0 \%$.

The average computation time for verification with images of size $232 \times 280$ pixels and using a Matlab implementation is about 2.5 seconds.

\section{Conclusions and Perspectives}

Hand vein verification is a promising technique for the authentication of an individual as the expected performance is very good. The performance we can expect is very good. This biometric modality is also difficult to copy that makes it a good candidate for many applications such as authentication method for e-banking operations.

In this paper, a complete biometric system based palm veins has been developed. We proposed an original method based on the use of SIFT descriptors for the enrollment and the verification steps. One of the main benefit of the proposed approach is that only one image is needed for the enrollment.

The efficiency of the proposed method is promising even if the test database used in the experiments is quite small. In the future, we intend to define a more significant database confirming our first preliminary results.

\section{References}

1. Im, S.-K., Park, H.-M., Kim, Y.-W., Han, S.-C., Kim, S.-o., Kang, C.-H.: An Biometric Identification System by Extracting Hand Vein Patterns. Journal of the Korean Physical Society 38(3) (March 2001)

2. Shahin, M., Badawi, A., Kamel, M.: Biometric Authentication Using Fast Correlation of Near Infrared Hand Vein Patterns. International journal of Biomedical sciences 2 (2007)

3. Zhang, Z., Ma, S., Han, X.: Multiscale Feature Extraction of Finger-Vein Patterns Based on Curvelets and Local Interconnection Structure Neural Network, Pattern Recognition. In: 18th International Conference on Pattern Recognition, vol. 4, pp. 145-148 (September 2006)

4. Watanabe, M., Endoh, T., Shiohara, M., Sasaki, S.: Palm vein authentication technology and its applications. In: Proceedings of the Biometric Consortium Conference, September 19-21 (2005)

5. Im, S.K., Choi, H.S., Kim, S.W.: A Direction-Based Vascular Pattern Extraction Algorithm for Hand Vascular Pattern Verification. ETRI J. 25(2), 101-108 (2008)

6. Wang, L., Leedham, G.: A Thermal Hand Vein Pattern Verification System. In: Singh, S., Singh, M., Apte, C., Perner, P. (eds.) ICAPR 2005. LNCS, vol. 3687, pp. 58-65. Springer, Heidelberg (2005)

7. Hong, L., Wan, Y., Jain, A.: Fingerprint Image Enhancement: Algorithm And Performance Evaluation. IEEE Transactions on Pattern Analysis and Machine Intelligence 20(8), $777-$ 789 (1998) 
8. Wang, J.-G., Yau, W.-Y., Suwandy, A., Sung, E.: Person recognition by fusing palmprint and palm vein images based on 'Laplacianpalm' representation. Pattern Recognition 41, 1531-1544 (2008)

9. Lowe, D.: Distinctive image features from scale-invariant key-points. International Journal of Computer Vision 60(2), 91-110 (2004)

10. Rosenberger, C., Brun, L.: Similarity-Based Matching for Face Authentication. In: International Conference on Pattern Recognition (ICPR) (2008)

11. Mikolajczyk, K., Schmid, C.: A performance evaluation of local descriptors. IEEE Transactions on Pattern Analysis and Machine Intelligence 27(10), 1615-1630 (2005) 